\title{
ОЦЕНКА РОЛИ ПОЛОВЫХ ГОРМОНОВ В РАЗВИТИИ ИНФАРКТА МИОКАРДА У ЖЕНЩИН В ПОСТМЕНОПАУЗЕ
}

\section{EVALUTION OF THE ROLE OF SEX HORMONES IN THE DEVELOPMENT OF MYOCARDIAL INFARCTION IN POSTMENOPAUSAL WOMEN}

\section{A. Beibalaeva M. Kudaev T. Gadjieva}

Summary. The article presents the results of a study of the level of sex hormones (estradiol and progesterone) and blood lipids in women with postmenopausal myocardial infarction and the relationship of these indicators. It was established that postmenopausal women with myocardial infarction have lower concentrations of estradiol and progesterone in comparison with the control group, and there are also changes in the blood lipid spectrum. The levels of estradiol and progesterone are inversely correlated with the concentration of cholesterol, low density lipoproteins and triglycerides. The highest statistically significant correlation was observed between the concentration of estradiol and triglycerides in blood serum $(R=-0.474 p=0.046)$. The revealed results confirm the point of view on the protective effect of sex hormones in the development of coronary artery disease.

Keywords: myocardial infarction, estradiol, progesterone, lipids, postmenopause.
Бейбалаева Амина Мусаибовна

Ассистент, Дагестанский государственный медицинский университет, Махачкала 89288350200@mail.ru

Кудаев Магомед Тагирович Д.м.н., профессор, Дагестанский государственный медицинский университет, Махачкала

kudaev54@mail.ru Гаджиева Тутубича Абдурахмановна

Д.м.н., профессор, Дагестанский государственный медицинский университет, Махачкала

tami2006@yandex.ru

Аннотация. В статье представлены результаты исследования уровня половых гормонов (эстрадиола и прогестерона) и липидов крови у женщин с инфарктом миокарда в постменопаузе и изучена взаимосвязь этих показателей. Установлено, что у женщин с инфарктом миокарда в постменопаузе отмечаются более низкие концентрации эстрадиола и прогестерона в сравнении с группой контроля, а также имеются нарушения в липидном спектре крови. Уровни эстрадиола и прогестерона имеют обратную корреляционную связь с концентрацией холестерина, липопротеидов низкой плотности и триглицеридов. Наибольшая статистически значимая корреляционная зависимость отмечена между концентрацией эстрадиола и триглицеридов в сыворотке крови $(R=-0,474, p=0,046)$. Выявленные результаты подтверждают точку зрения о протективном влиянии половых гормонов в развитии ИБС.

Ключевые слова: инфаркт миокарда, эстрадиол, прогестерон, липиды, постменопауза.

функцию эндотелия, препятствуя ремоделированию сосудистой стенки [7]. Значимость прогестерона в снижении риска ИБС заключается в его влиянии на процессы реполяризации в миокарде путем модуляции синтеза оксида азота [8] и способности уменьшать выраженность воспаления [9], что важно для реализации защитного эффекта против ишемического поражения. В то же время, за последние два десятилетия, кардиопротективные эффекты эстрогенов были поставлены под сомнение. В таких исследованиях, какWomen's Health Initiative [10] и The Heart and Estrogen- progestin Replacement Study [11] назначение заместительной гормональной терапии эстрогенами показало увеличение риска сердечно - сосудистых событий, что было связано с их тромбогенными эффектами.

Отсутствие единой точки зрения на вопрос о влиянии половых гормонов на риск развития ИБС у женщин в постменопаузе делает проведение новых исследований 
Таблица 1. Лабораторно - инструментальная характеристика женщин в постменопаузе с диагнозом ИМ (І группа)

\begin{tabular}{|l|l|}
\hline Показатель & Женщины \\
\hline ИмТ* & $29,9 \pm 3,8$ \\
\hline Глюкоза, ммоль/л & $6,8 \pm 2,2$ \\
\hline Гемоглобин, г/л & $122,0 \pm 6,4$ \\
\hline Тропонин І, нг/мл & $1,21 \pm 1,8$ \\
\hline Креатинин, мкмоль/л & $89,0 \pm 3,8$ \\
\hline СКФ ${ }^{* *}$ мл/мин/1,73, м² & $72,0 \pm 8,5$ \\
\hline ЧСС & $82,0 \pm 5,1$ \\
\hline Среднее АД, мм.рт.ст. & $135 \pm 7,2$ \\
\hline Фракция выброса Лж***,\% & $52 \pm 4,4$ \\
\hline
\end{tabular}

*ИМТ — индекс массы тела, ${ }^{* *}$ СКФ - скорость клубочковой фильтрации, ***ЛЖ - левый желудочек.

в данном направлении актуальным. Особенный интерес, на наш взгляд, представляет изучение баланса половых гормонов в постменопаузе у женщин с подтверждённым диагнозом инфаркта миокарда (ИМ).

\section{Шель исслеАования}

Провести сравнительный анализ и оценить взаимосвязь уровня половых гормонов и показателей липидного спектра у женщин с ИМ в постменопаузе.

\section{Материалы и метолы}

В исследование были включены 36 женщин (I группа), которые проходили лечение в инфарктном отделении Республиканской клинической больницы скорой медицинской помощи г. Махачкалы, в период с 1.03.2018 г. по 30.06.2019 г. с подтверждённым диагнозом ИБС, инфаркт миокарда. Возраст обследованных составил $59,2 \pm 0,7$ лет. Все женщины I группы находились в постменопаузе, возраст наступления которой составил в среднем $53,0 \pm 8,1$ г. В группу контроля вошли 20 здоровых женщин в постменопаузе (II группа), средний возраст составил $58,1 \pm 0,6$ лет. Возраст наступления менопаузы во II группе

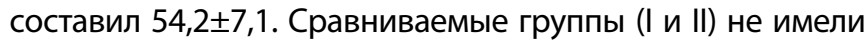
статистически значимых различий по возрасту ( $>>0,05)$.

На всех обследованных заполнялась индивидуальная регистрационная карта пациента, в обеих группах проводили стандартные лабораторные исследования, в том числе определяли уровни липидов (холестерина $(X C)$, липопротеидов высокой плотности (ЛПВП), липопротеидов низкой плотности (ЛПНП), триглицеридов (ТГ)). Методом иммуноферментного анализа оценивался уровень половых гормонов (эстрадиола, прогестерона) в нг\мл.

Инструментальные методы обследования включали ЭКГ, ЭхоКГ, коронарографию (КАГ). КАГ проводилась на ангиографической установке Innova 4100 (GE, Германия). Оценивали варианты поражения коронарных артерий (КА): одно-, двух- и трехсосудистое. Сужение КА расценивали как гемодинамически значимое при стенозе $\geq$ $60 \%$.

Для оценки статистической связи между показателями уровня половых гормонов у женщин с ИМ и показателями липидного спектра (ХС, ХС ЛПНП, ХС ЛПВП, ТГ) применили метод корреляционного анализа по Спирмену. Корреляционная связь между величинами оценивалась как слабая при значениях коэффициента корреляции менее 0,3, от 0,3 до 0,69- как связь средней силы, при коэффициенте корреляции более 0,7 - как сильная.

Статистическая обработка проводилась с использованием пакета прикладных программ «Statistica 6.0». Для оценки достоверности различий между средними значениями показателей сопоставляемых групп ( $\mathrm{M} \pm \mathrm{m}$, где M - среднее значение, $\mathrm{m}$ - стандартная ошибка средней) применяли t-критерий Стьюдента. Достоверными считали различия на уровне значимости $\mathrm{p}<0,05$.

\section{Результаты}

Женщины в постменопаузе $(n=36)$, госпитализированные с диагнозом ИБС ИМ, проходили стандартные лабораторно-инструментальные исследования на базе инфарктного отделения Республиканской клинической больницы скорой медицинской помощи г. Махачкалы. Данные представлены табл. 1.

Инфаркт миокарда с формированием зубца Q был диагностирован у 15(41,7\%) женщин, не Q- инфаркт у 21 (58,3\%).

Для определения распространенности поражения KА, у обследованных женщин с ИМ (I rp, n=36) проведен анализ коронарограмм, который показал, что атеро- 
Таблица 2. Концентрации половых гормонов (нг/мл) в сыворотке крови у женщин в постменопаузе с инфарктом миокарда (I) в сравнении с группой контроля (II)

\begin{tabular}{|l|l|l|l|}
\hline $\begin{array}{l}\text { Показатель } \\
\text { (нг/мл) }\end{array}$ & $\begin{array}{l}\text { I группа } \\
\text { (больные ИМ, } \mathbf{n = 3 6 )}\end{array}$ & II (группа контроля) (n=20) & P \\
\hline Эстрадиол & $44,74 \pm 3,17$ & $54,33 \pm 2,7$ & $P<0,05$ \\
\hline Прогестерон & $0,67 \pm 0,43$ & $0,75 \pm 0,61$ & $P>0,05$ \\
\hline
\end{tabular}

Таблица 3. Уровень липидов крови у женщин с инфарктом миокарда (I гр.) и в группе контроля (II гр.)

\begin{tabular}{|l|l|l|l|}
\hline (ммоль/л) & группа (женщины с иМ, $\mathbf{n = 3 6 )}$ & (здоровые лица, $\mathbf{n = 2 0}$ & \\
\hline Общий холестерин & $, 8 \pm 0,1$ & $, 7 \pm 0,10$ &, 131 \\
\hline Триглицериды & $, 4 \pm 0,18^{*}$ & $, 6 \pm 0,32$ &, 022 \\
\hline ХС-ЛПНП & $, 4 \pm 0,18^{*}$ & $, 2 \pm 0,72$ &, 047 \\
\hline ХС-ЛПВП & $, 0 \pm 0,19$ & $, 1 \pm 0,03$ &, 119 \\
\hline
\end{tabular}

* - различия статистически значимы

склеротический процесс носил распространенный характер, чаще имело место многососудистое поражение коронарного русла - в 56,6\% случаев. Изолированное поражение одной из коронарных артерий наблюдалось лишь в 20,7\%, две коронарные артерии были поражены у 22,7\% женщин. Правый тип коронарного кровообращения (ККО) наблюдался в 91,3\% случаев, сбалансированный в 8,7\%. Левый тип ККО у обследованных женщин не был выявлен. Полученные результаты согласуются с превалирующим большинством научных данных по данному вопросу [12], хотя имеется и противоположная точка зрения. Так, в исследовании А.Ю. Анисенко, 2006 г. утверждается о доминировании сбалансированного типа ККО у женщин с ИБС.

По данным когортных исследований, большинство факторов риска ИБС и ИМ общие для мужчин и женщин [13], но существует уникальный женский фактор риска развития и прогрессирования ССЗ - естественное физиологическое состояние женского организма - менопауза.

Для изучения роли половых гормонов в развитии ИМ у обследованных женщин с ИМ(Ігр.; $\mathrm{n}=36$ ) проведена оценка концентраций эстрадиола и прогестерона в сыворотке крови. В Группу контроля были включены 20 здоровых женщин без признаков ИБС (II гр.; $\mathrm{n}=20)$. Результаты представлены в табл. 2.

Как видим, у женщин в постменопаузе с подтверждённым диагнозом ИМ (I гр.) уровень половых гормонов был ниже в сравнении с группой контроля (II гр.). Концентрация эстрадиола в I гр. на 21,3\% была статистически значимо $(p<0,05)$ меньше, чем в контрольной (II гр.). Концентрация прогестерона у больных женщин (I гр.) была ниже, чем в группе контроля на 10,7\%, одна- ко выявленные различия не имели статистической значимости ( $>0,05)$. Полученные данные подтверждаются рядом научных публикаций, в которых установлена защитная роль половых гормонов $[4,14]$.

В табл. 3 представлены результаты сравнительного анализа показателей липидного спектра в группе больных женщин с ИМ (І гр.) в сравнении с группой контроля (II гр.).

Как свидетельствуют данные табл. 3, уровни ХС, ТГ, лПНП были выше в группе больных женщин с ИМ (I гр., $\mathrm{n}=36)$ в сравнении с группой контроля, при этом статистически значимое превышение отмечалось по уровню ТГ $(p=0,013)$ и ХС - ЛПНП $(p=0,026)$. Выявленные особенности согласуются с результатами научных исследований, в которых определена важная роль повышения уровня ТГ в патогенезе женской ИБС $[15,16,17]$.

Среди множества предполагаемых механизмов, с помощью которых эндогенные эстрогены могут защищать от СС3, выделяют положительное влияние эстрадиола на липидный профиль плазмы $[18,19]$. В связи с этим в рамках настоящего исследования проведен корреляционный анализ между уровнем эстрадиола и показателями липидного обмена (XС, ХС ЛПНП, ХС ЛПВП) в группе обследованных больных (I гр; $n=36$ ). Установлено, что показатели эстрадиола в сыворотке крови у женщин в постменопаузе с ИМ имели статистически значимую обратную корреляционную связь с концентрациями ТГ $(r=-0,47 ; p=0,046)$. Между показателями эстрадиола и холестерина в сыворотке крови $(r=-0,24 ; p=0,064)$, а также между показателями эстрадиола и значениями ХС ЛПНП ( $r=-0,26 ; p=0,072)$ корреляционная зависимость также была отрицательной, но слабой и статистически недостоверной. Корреляционная зависимость 
между значениями прогестерона у женщин с ИМ и изучаемыми показателями липидного обмена была слабой, отрицательной, статистически незначимой ( $p>0,05$. Установление статистически значимой средней силы обратной корреляционной зависимости между уровнем эстрадиола и триглицеридов в сыворотке крови у обследованных женщин с ИМ, находящихся в постменопаузе, не позволяет утверждать однозначно о наличии причинно-следственной связи между этими показателями. Возможно, что дальнейшие исследования выявленных закономерностей на более обширном статистическом материале позволят сделать более основательные выводы.
На основании результатов настоящего исследования были сделаны следующие выводы:

1. У женщин с ИМ в постменопаузе отмечался более низкий уровень половых гормонов (эстрадиол, прогестерон) в сравнении с группой контроля;

2. У женщин с ИМ в постменопаузе имелись нарушения в липидном спектре крови - статистически значимое снижение уровня триглицеридов и ХC ЛПНП в сравнении с группой контроля $(p<0,05)$;

3. Показатели эстрадиола в сыворотке крови у женщин в постменопаузе с ИМ имели статистически значимую обратную корреляционную связь с концентрациями ТГ $(r=-0,47 ; p=0,046)$;

\section{ЛИТЕРАТУРА}

1. Perk J., De B. G., Gohlke H., et al. European Guidelines on cardiovascular disease prevention in clinical practice (version 2012). The Fifth Joint Task Force of the European Society of Cardiology and Other Societies on Cardiovascular Disease Prevention in Clinical Practice (constituted by representatives of nine societies and by invited experts) // Eur Heart J.— 2012.— № 33.—P.1635-1701.

2. Dubey R.K., Imthurn B., Zacharia L.C., Jackson E. K. Hormone replacement therapy and cardiovascular disease: what went wrong and where do we go from here?// Hypertension. - 2004.— № 44.—P.789-795.

3. Orshal J.M., Khalil R. A. Gender, sex hormones, and vascular tone //Am J Physiol Regul Integr Comp Physiol.— 2004.— № 286.—P. R233-R249

4. Benn M., Voss S. S., Holmegard H. N., Jensen G. B., Tybjaerg-Hansen A., Nordestgaard B. G. Extreme concentrations of endogenous sex hormones, ischemic heart disease, and death in women // Arterioscler Thromb Vasc Biol.— 2015.— № 35.— P.471-7.

5. Baler L., Meldrum K., Wang M. et al. The role of estrogen incardiovasculardisease // J Surg Res. — 2003.— № 115(2). — P.325-44.

6. FischerM., Baessler A., Schunkert H. Renin angiotensin system and gender differences in the cardiovascular system // Cardiovasc Res. — 2002.— № 53(3). P.672-7.

7. WengerN. Coronary heart disease: an older woman'smajor health risk // BMJ.— 1997.— № 315(7115). — P.1085-90.

8. Losert W., Casals-Stenzel J., Buse M. Progestogens with antimineralocorticoid activity // Arzneimittelforschung. — 1985. — № 35(2). — P.459-71

9. Dhote, V.V., Balaraman R. Gender specific effect of progesterone on myocardial ischemia/ reperfusion injury in rats // Life Sci.— 2007.— № 81(3). -P.188-97.

10. Rossouw J.E., Anderson G. L., Prentice R. L., et al.Risks and Benefits of Estrogen Plus Progestin in Healthy Postmenopausal Women: Principal Results From the Women's Health Initiative Randomized Controlled Trial // JAMA. — 2002. - № 288 (3). — P.321-33.

11. HulleyS., GradyD., BushT., FurbergC., HerringtonD., RiggsB, E. Randomized Trial of Estrogen Plus Progestin for Secondary Prevention of Coronary Heart Disease in Postmenopausal Women. Heart and Estrogen/progestin Replacement Study (HERS) Research Group //JAMA. — 1998. — № 280(7). — P.605-13

12. Koşar P, Erqun E, Öztürk C, et al. Anatomic variations and anomalies of the coronary arteries: 64-slice CT angiographic appearance // Diagn Interv Radiol.— 2009. № $15(4)$. - P.275-83.

13. Гиляревский С. Р. Азбука вторичной профилактики ишемической болезни сердца. Тактика и стратегия лечения больных, перенесших острый коронарный синдром или инфаркт миокарда // Сердце. — 2002.-№ 1(1).—C. 34-37

14. Colditz G.A., WillettW.C., Stampfer M. J., Rosner B., Speizer F. E., Hennekens C. H. Menopause and the risk of coronary heart disease in women// N Engl J Med.—1987.— № 316. — P.1105-1110.

15. Masi S., Lautamaki R., Guiducci L. et al. Similar patterns of myocardial metabolism and perfusion in patients with type 2 diabetes and heart disease of ischaemic and nonischaemic origin // Diabetologia.—2012.— № 55(9).—P.2494-500

16. Gori T., Fineschi M. Two coronary «orphan» diseases in search of clinical consideration: coronary syndromes $x$ and y // Cardiovasc Ther. — 2012. - № 30(2). —P. e58-65

17. Nakazato R., Dey D., Cheng V.Y. et al. Epicardial fat volume and concurrent presence of both myocardial ischemia and obstructive coronary artery disease. // Atherosclerosis. - 2012. — № 221(2).—P.422-6.

18. Мартынов А.И. и др. Особенности липидного профиля у женщин в период менопаузы, как фактор риска сердечно — сосудистых заболеваний // Рос. мед. вестн. - 2003. - Т. 2.- C. 22-27.

19. Сметник В. П. Системные изменения у женщин в климактерии // Рус. мед. журн. — 2001.—Т.9 (9). — С. 354-358. 